Analysis of localization phenomena in weakly interacting disordered lattice gases

This content has been downloaded from IOPscience. Please scroll down to see the full text.

2006 New J. Phys. 8230

(http://iopscience.iop.org/1367-2630/8/10/230)

View the table of contents for this issue, or go to the journal homepage for more

Download details:

IP Address: 194.95.157.145

This content was downloaded on 05/04/2017 at 14:34

Please note that terms and conditions apply.

You may also be interested in:

Experimental study of the transport of coherent interacting matter-waves in a 1D random potential induced by laser speckle

D Clément, A F Varón, J A Retter et al.

$\underline{\text { Disorder-induced trapping versus Anderson localization in Bose-Einstein condensates expanding in }}$ disordered potentials

L Sanchez-Palencia, D Clément, P Lugan et al.

Dilute Bose gas with correlated disorder: a path integral Monte Carlo study

$S$ Pilati, S Giorgini, M Modugno et al.

Numerical study of one-dimensional and interacting Bose--Einstein condensates

Eric Akkermans, Sankalpa Ghosh and Ziad H Musslimani

Anderson localization in Bose-Einstein condensates

Giovanni Modugno

Regimes of classical transport of cold gases in a two-dimensional anisotropic disorder

L Pezzé, M Robert-de-Saint-Vincent, T Bourdel et al.

Correlation function of weakly interacting bosons in a disordered lattice

B Deissler, E Lucioni, M Modugno et al.

Cold atoms in the presence of disorder

Boris Shapiro 


\title{
Analysis of localization phenomena in weakly interacting disordered lattice gases
}

\author{
T Schulte $^{1}$, S Drenkelforth ${ }^{1}$, J Kruse ${ }^{1}$, R Tiemeyer $^{1}$, K Sacha ${ }^{2,3}$, \\ $\mathbf{J}$ Zakrzewski $^{2,3}$, M Lewenstein ${ }^{4,5,6}$, W Ertmer ${ }^{1}$ and $\mathbf{J} \mathbf{J}$ Arlt $^{1}$ \\ ${ }^{1}$ Institut für Quantenoptik, Leibniz Universität Hannover, Welfengarten 1, \\ D-30167 Hannover, Germany \\ 2 Instytut Fizyki imienia Mariana Smoluchowskiego, Uniwersytet Jagielloński, \\ PL-30-059 Krakow, Poland \\ ${ }^{3}$ Marc Kac Complex Systems Research Center, Uniwersytet Jagielloński, \\ PL-30-059 Krakow, Poland \\ ${ }^{4}$ Institut für Theoretische Physik, Leibniz Universität Hannover, \\ D-30167 Hannover, Germany \\ ${ }^{5}$ ICFO-Institut de Ciéncies Fotóniques, 08034 Barcelona, Spain \\ E-mail: schulte@iqo.uni-hannover.de
}

New Journal of Physics 8 (2006) 230

Received 17 July 2006

Published 9 October 2006

Online at http://www.njp.org/

doi:10.1088/1367-2630/8/10/230

\begin{abstract}
Disorder plays a crucial role in many systems particularly in solid state physics. However, the disorder in a particular system cannot usually be chosen or controlled. We show that the unique control available for ultracold atomic gases may be used for the production and observation of disordered quantum degenerate gases. A detailed analysis of localization effects for two possible realizations of a disordered potential is presented. In a theoretical analysis, clear localization effects are observed when a superlattice is used to provide a quasiperiodic disorder. The effects of localization are analysed by investigating the superfluid fraction and the localization length within the system. The theoretical analysis in this paper paves a clear path for the future observation of Anderson-like localization in disordered quantum gases.
\end{abstract}

${ }^{6}$ Also at Institució Catalana de Recerca i Estudis Avançats. 


\section{Contents}

1. Disordered lattice potential 3

1.1. Experimental results. . . . . . . . . . . . . . . . 3

1.2. Theoretical analysis . . . . . . . . . . . . . . . 6

2. Superlattice potential $\quad 8$

2.1. Screening due to interaction. . . . . . . . . . . . . . . 9

2.2. Analysis of localization . . . . . . . . . . . . . . . . . 10

2.3. Analysis of superfluidity. . . . . . . . . . . . . . . . . . . . . . . 12

2.4. Detection of localization . . . . . . . . . . . . . . . 13

3. Conclusion 15

Acknowledgments $\quad 15$

$\begin{array}{ll}\text { References } & 15\end{array}$

In condensed matter or statistical physics disorder is typically neither avoidable nor controllable. It is known to lead to spectacular phenomena, such as Anderson localization [1,2]. In this case, the interference of waves scattered on random impurities or defects is responsible for localization. In the so-called weak localization regime coherent backscattering may be regarded as a precursor for Anderson localization [3]. For a recent treatment of light waves scattered by atomic ensembles, see e.g. [4].

Cold atomic gases in magnetic and optical potentials offer the unique possibility to introduce well-controlled disorder to the system. For this purpose several methods have been proposed to create disordered, or quasi-disordered potentials. These proposals include the use of speckle radiation [5]-[7], incommensurable optical lattices [7]-[11] and the interaction with impurity atoms [12]. The unique control provided by these methods should enable novel studies of disorder induced effects, inaccessible to other systems. In addition disorder appears also naturally close to the surface of atom chips [13] and leads to a fragmentation of Bose-Einstein condensates (BEC).

The first experimental attempts to systematically study the effects of disorder on BEC used a speckle potential. The Florence group [14] observed the apparent fragmentation of the condensate into pieces in the presence of such a random potential, resulting in characteristic stripes in the time-of-flight density profiles. Another experiment, performed simultaneously in Florence [15] and in Orsay [16], showed that in the presence of the random potential the expansion of the condensate is strongly inhibited. A plausible scenario for this suppression of transport given in [16]-[18] notes that the transport stops when the BEC encounters a random potential modulation of sufficient height. This explanation is purely classical but it is supported by simulations of the Gross-Pitaevskii equation (GPE).

Another disorder configuration was realized in our group by combining a onedimensional (1D) optical lattice and a random potential [19]. This potential configuration was designed to mimic the situation first envisaged by Anderson [2]. However, it was shown that, contrary to first expectations, one cannot observe Anderson localization in this experiment due to interaction effects and the length scale of the employed disorder potential. We discuss this situation in more detail in the following sections. 
The onset of the Bose-glass phase has recently been observed [20] for ultracold atoms in the strongly interacting regime as suggested by [7]. For more details on strongly interacting bosonic systems, we refer the reader to the seminal discussion in [21]. We also note that mixtures of fermions and bosons in random optical potentials are of considerable interest [22]. One component in such a mixture may in fact serve as the disorder in the system [23]. Here, we concentrate solely on the weakly interacting case for bosons and investigate the circumstances for the appearance of Anderson-like localization.

The paper is organized as follows. In the first part, we describe the experimental realization of a weakly interacting lattice gas with a superimposed slowly varying disorder potential and discuss the ground state properties of such a system. We observe a classical fragmentation of the condensate which is confirmed by a theoretical analysis based on the GPE but no signature of Anderson localization is found.

Therefore we theoretically investigate another type of disorder which has a shorter correlation length in the second part. Following earlier suggestions [7, 9, 10] we consider the ground state of the condensate in the presence of a pseudorandom potential introduced by additional optical lattices of different wavelengths. Such a potential is called a superlattice $[7,9,10]$. We discuss the influence of nonlinear interactions on the occurrence of localization phenomena in this system.

\section{Disordered lattice potential}

\subsection{Experimental results}

Despite a variety of possible realizations of disorder, one method is particularly straightforward to implement in current experimental setups. It consists of projecting a disordered optical dipole potential on to the atomic sample. Following the theoretical suggestion [7] such realizations have recently been used to investigate the effects of disorder on the ground state $[14,19]$ and on the dynamics of weakly interacting BECs [14]-[16].

We perform our experiments with ${ }^{87} \mathrm{Rb} \mathrm{BECs}$ in an elongated magnetic trap. After laser cooling and trapping cold atoms are loaded into a cloverleaf magnetic trap with axial and radial frequencies of $\omega_{x}=2 \pi \times 14 \mathrm{~Hz}$ and $\omega_{\perp}=2 \pi \times 200 \mathrm{~Hz}$, respectively. These atoms are evaporatively cooled to quantum degeneracy resulting in a final number of condensed atoms between $1.5 \times 10^{4}$ and $8 \times 10^{4}$.

To carry out experiments in a disordered lattice configuration we use two optical dipole potentials, a 1D lattice and the disordered potential. Both are derived from a Ti : Sa laser operating at a wavelength of $\lambda=825 \mathrm{~nm}$. Acousto-optic modulators are used to control the intensity of each dipole potential and optical fibres are employed to deliver the light beams to the experiment.

The optical lattice is created by retro-reflection of a laser beam along the axial direction of the magnetic trap. The depth of the optical lattice is typically set to $6.5 E_{\mathrm{r}}$. The recoil energy $E_{\mathrm{r}}$ is given by $E_{\mathrm{r}}=\hbar^{2} k^{2} / 2 m$, where $m$ denotes the atomic mass and $k$ corresponds to the wavenumber of the optical lattice. The detection system is used to image the axial position of the atomic cloud as well as the beam waist. This allows for precise positioning of the beam waist with respect to the atomic sample.

The disorder potential is created by illuminating a randomly structured chrome substrate with a laser beam. The partially transmitting substrate is imaged onto the atomic sample from 


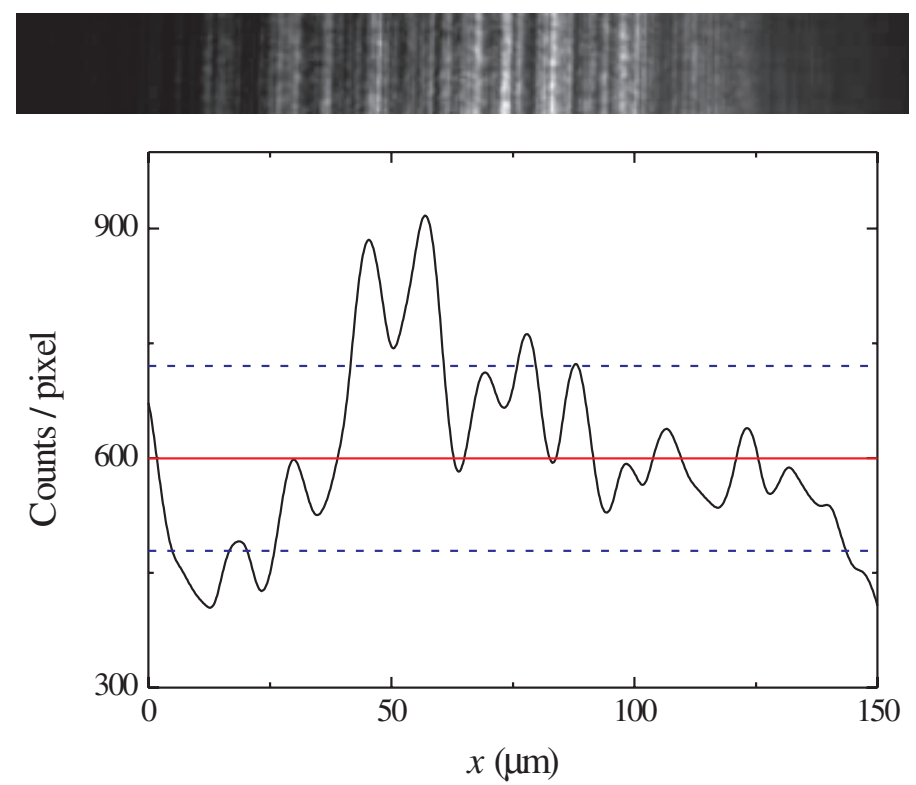

Figure 1. Typical intensity distribution of the disordered optical dipole potential. An image of the intensity distribution is shown at the top. The intensity variation in a small region corresponding to the size of the sample is shown below.

a direction orthogonal to the optical lattice. Due to the resolution of the imaging system, the minimal structure size of the dipole potential is limited to a few micrometres. Figure 1 shows an image of the disorder potential at the position of the atomic cloud. We define the depth of the disorder potential $V_{\Delta}$ as twice the standard deviation of the dipole potential, analogously to [14]. Figure 2 shows the calculated autocorrelation function

$$
g(L)=\frac{\left\langle V_{\mathrm{dis}}(x) V_{\mathrm{dis}}(x+L)\right\rangle}{\left\langle V_{\mathrm{dis}}(x)\right\rangle\left\langle V_{\mathrm{dis}}(x+L)\right\rangle} .
$$

for a typical realization of the disordered potential. In (1) the brackets denote the average over position and $V_{\text {dis }}(x)$ represents the disordered potential. Figure 2 shows that the correlation length of the potential decays on a typical length scale of $10 \mu \mathrm{m}$.

After the production of the BEC, our experiments are performed as follows. The optical lattice is ramped to its final depth within $60 \mathrm{~ms}$. Subsequently the disorder potential is increased to its final depth within another $60 \mathrm{~ms}$ followed by a hold time of $20 \mathrm{~ms}$. Finally all potentials are switched off and the atomic density distribution is measured after $20 \mathrm{~ms}$ of free expansion using absorption imaging.

In the expanded atomic density distributions, we observe significant irregular modulations which depend on the strength of the disordered potential. To quantify these modulations, we fit the central peak of the expanded lattice gas with an inverted parabolic distribution and calculate the standard deviation $\sigma$ of the measured density profile from this fit. Figure 3 shows the dependence of the standard deviation on the depth of the disordered potential. Clearly the deviation from the unperturbed parabolic distribution grows with increased disorder strength. 


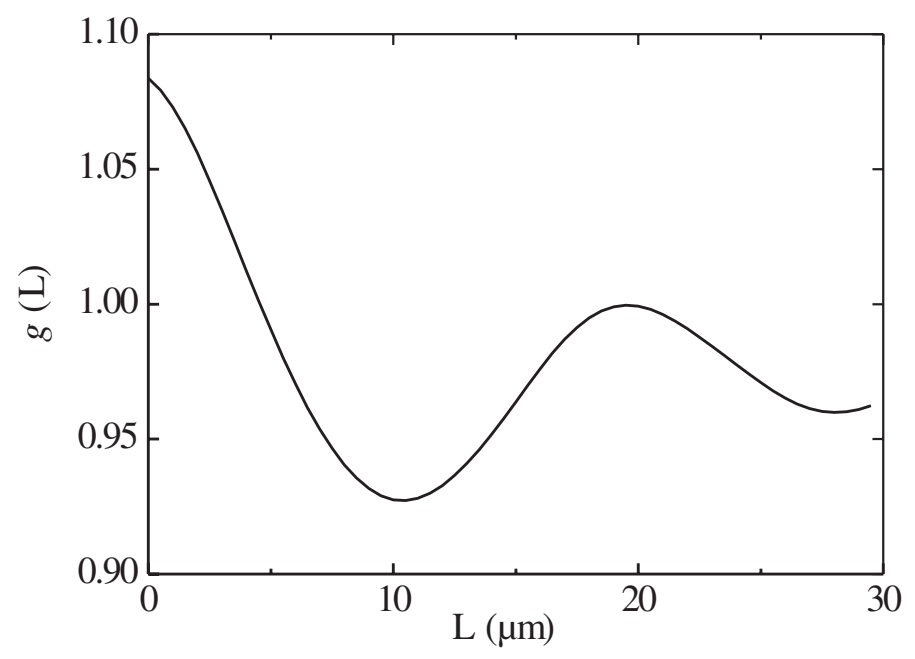

Figure 2. Autocorrelation function for a typical realization of the disordered potential.

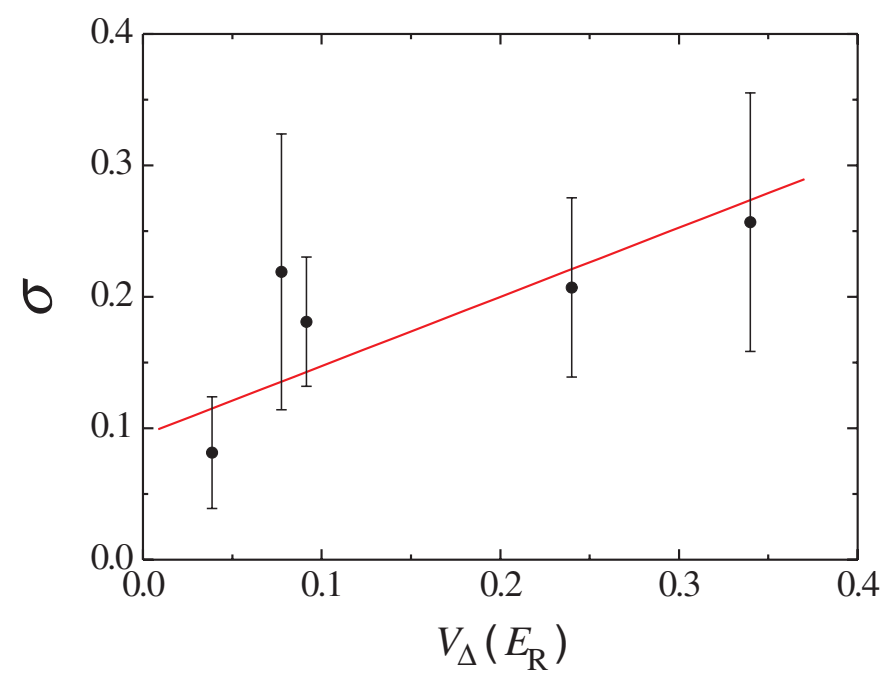

Figure 3. Standard deviation $\sigma$ of the observed axial density distribution from an inverted parabola after $20 \mathrm{~ms}$ of free expansion, depending on the disorder strength. The red line is a linear fit to the data which serves as a guide to the eye.

To further investigate the expansion of the disordered lattice gas, we extract the axial size of the central momentum peak from the fits of the density with an inverted parabolic distribution. Figure 4 shows the resulting sizes for four different configurations of the combined potential as a function of the atom number.

The red curve shows the theoretical prediction based on the Thomas-Fermi approximation and subsequent self-similar expansion [24] for a confinement in the magnetic trap only. The blue curve shows an estimate for the expansion of a lattice gas in the absence of disorder. It is obtained by using the increased axial size of the cloud due to the presence of the optical lattice [25] as a starting point for the self-similar expansion. 


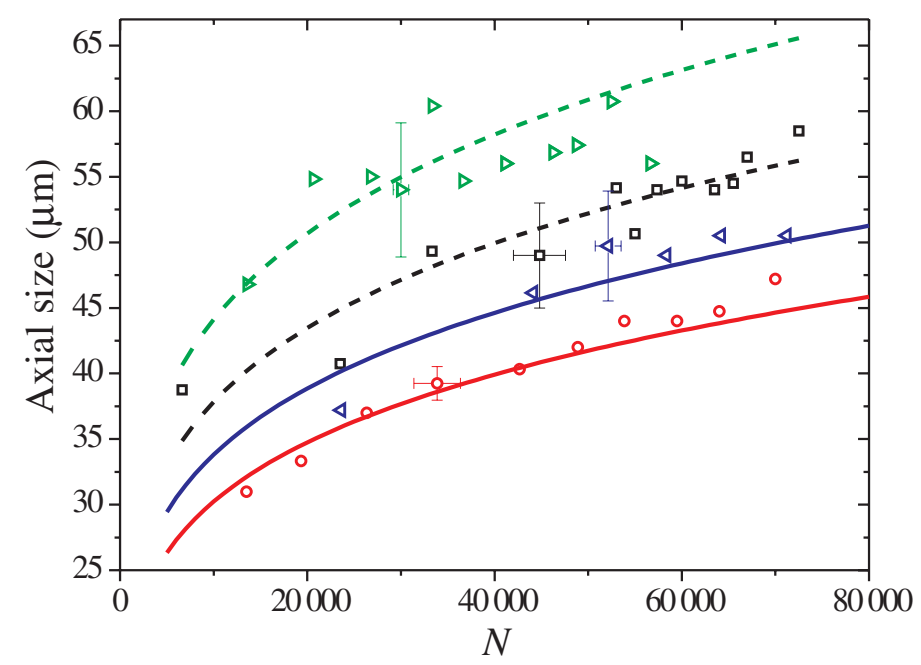

Figure 4. Size of the central peak after $20 \mathrm{~ms}$ of free expansion versus the number of atoms. The clouds are released from the following potentials: magnetic trap (red o), magnetic trap and disorder (black $\square$ ), magnetic trap and optical lattice (blue $\triangleleft$ ), magnetic trap and disorder and lattice (green $\triangleright$ ). The lines correspond to a theoretical prediction (see text). The lattice depth is $6.5 E_{\mathrm{r}}$ and the disorder has a depth of $0.1 E_{\mathrm{r}}$.

The addition of the disorder potential yields a more surprising result. Despite its very small potential depth, the additional axial confinement due to the disorder potential leads to a significant increase of the axial size after expansion. The theoretical analysis below shows that the disordered potential induces strong deviations of the density profile from the parabolic envelope. Therefore significant deviations from the self similar expansion are expected for disordered gases. According to figure 3, these deviations produce irregular density modulations and can lead to pronounced changes in the widths of the expanded clouds. The explicit form of the expanded density distribution, however, depends strongly on the exact realization of the disordered potential. We have used a 3D numerical simulation to check the expansion of the disordered gas in the absence of the optical lattice. Depending on the disorder potential used, the simulation confirms the observed behaviour qualitatively.

\subsection{Theoretical analysis}

In the system considered here, the disorder potential is imposed along one axis of the cloud. Hence a 1D GPE is used to describe the basic properties of the system and to analyse parameter regimes where localization phenomena can be observed. The GPE provides an appropriate description of the system as long as depletion effects are small. We have estimated the number of atoms depleted from the condensate wave-function within the Bogoliubov theory for the optical lattice potential used in the experiment. For a total particle number of $10^{5}$ atoms the fraction of depleted atoms is less than $1 \%$.

The 1D GPE used in our simulations is given by

$$
\mathrm{i} \partial_{t} \phi=\left[-\frac{\partial_{x}^{2}}{2}+\frac{x^{2}}{2}+V_{0} \cos ^{2}(k x)+V_{\text {dis }}(x)+g|\phi|^{2}\right] \phi,
$$


where we have adopted harmonic oscillator units, i.e. $\hbar \omega_{x}, \sqrt{\hbar / m \omega_{x}}$ and $1 / \omega_{x}$ as energy, length and time units. The depth of the optical lattice is given by $V_{0}$ and $V_{\text {dis }}(x)$ denotes the disordered potential. In the 3D case, the coupling constant is given by $g_{3 \mathrm{D}}=4 \pi \hbar^{2} a / m$, where $a$ denotes the $s$-wave scattering length. For the 1D simulations, the coupling constant $g$ is chosen such that the Thomas-Fermi radius of the 1D gas equals the axial Thomas-Fermi radius in the 3D trap.

To simulate the experimental situation, the wavelength of the lattice is set to $\lambda=825 \mathrm{~nm}$. Since the harmonic trap and the disorder potential change on a length scale much greater than the lattice spacing and the condensate healing length, $l=1 / \sqrt{8 \pi n a}$ (where $n$ is the condensate density) we eliminate the lattice potential in the GPE by applying the so-called effective mass analysis [26].

Within this analysis the GPE (2) is replaced by an equation where the optical lattice potential is absent but the mass of a particle and the coupling constant are corrected. We assume that the ground state solution of the GPE has the form

$$
\phi_{0}(x)=\sqrt{\mathcal{N}} f(x) u_{0}(x)
$$

Here $u_{0}(x)$ denotes the Bloch function corresponding to the ground state of the Schrödinger equation with the optical lattice potential, $f(x)$ is an envelope function and $\mathcal{N}$ is a normalization factor. Substituting the ansatz (3) into equation (2) leads to the equation

$$
\mu^{*} f(x)=\left[-\frac{\partial_{x}^{2}}{2 m^{*}}+\frac{x^{2}}{2}+V_{\mathrm{dis}}(x)+g^{*}|f(x)|^{2}\right] f(x),
$$

where $m^{*}$ and $g^{*}$ are the effective mass and the effective coupling constant, respectively. For an optical lattice depth of $6.5 E_{\mathrm{r}}$, the effective parameters are

$$
m^{*}=2.56 m, \quad g^{*}=1.66 g .
$$

For the total number of $N=10^{5}$ atoms (that implies $g=1800$ ), the values of the effective parameters suggest that one can employ the Thomas-Fermi approximation and neglect the kinetic energy term. Then the solution of equation (4) is

$$
|f(x)|^{2}=\frac{\mu^{*}-x^{2} / 2-V_{\mathrm{dis}}(x)}{g^{*}},
$$

where $\mu^{*}$ is determined from the normalization condition

$$
\int|f(x)|^{2} \mathrm{~d} x=1
$$

In figure 5, we show a comparison of the ground state solution of the full GPE (2) and the solution of the effective mass approach obtained within the Thomas-Fermi approximation. The squared overlap of these solutions is greater than 0.99 and hence they are practically identical.

This analysis shows that the effect of a slowly varying disorder potential applied to BEC in the lattice potential can be described within the Thomas-Fermi approximation. The condensate density is modulated by the slowly varying disorder and consequently no Anderson localization is present. 


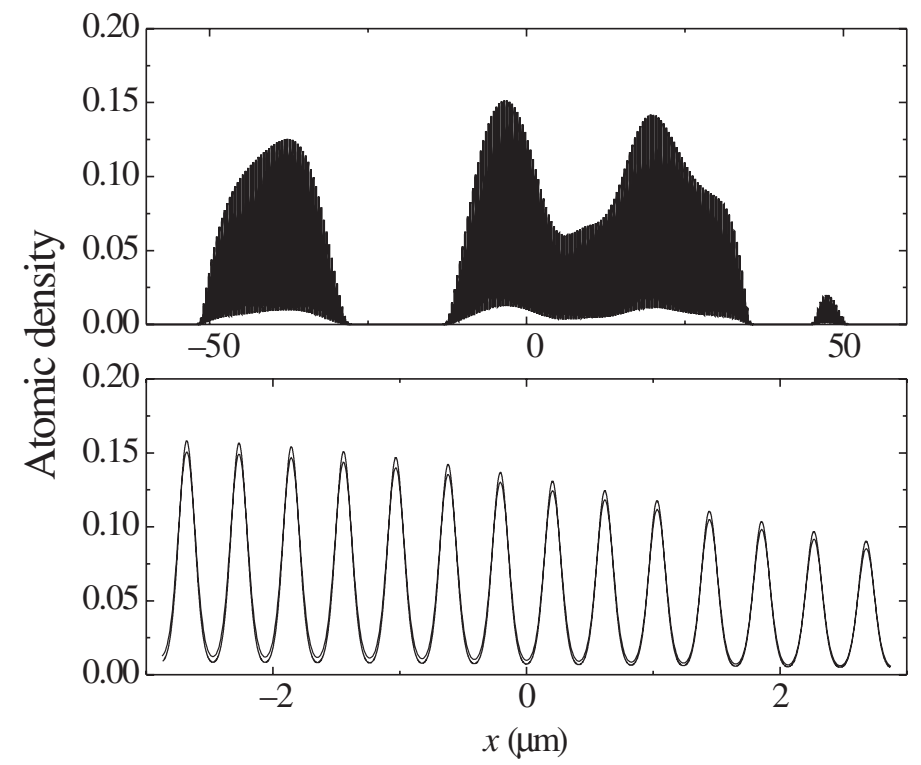

Figure 5. Top panel: ground state solution of the GPE for a condensate in the combined potential of the magnetic trap $\left(\omega_{x}=2 \pi \times 14 \mathrm{~Hz}\right)$, optical lattice and disorder potential. Bottom panel: central part of the ground state solution of the GPE (solid line) and the corresponding solution within the effective mass approach (dashed line). The depth of the optical lattice is $6.5 E_{\mathrm{r}}$, while the depth of the disorder potential $0.7 E_{\mathrm{r}}$.

These results thus bear similarity to the experiments performed in the absence of an optical lattice [14] where a fragmentation of the BEC is induced by the disorder potential.

\section{Superlattice potential}

The above analysis suggests that it is necessary to introduce a disorder that changes on a length scale smaller than the healing length to enter a regime where localization effects can be observed. To overcome the experimental difficulties of imposing such a truly random potential the use of pseudorandom potentials has been suggested [7,9].

These pseudorandom potentials can be formed by one or more additional optical lattices creating a so-called superlattice. Since cold atomic gases present a finite sized system, a suitably chosen pseudorandom potential can provide the desired disorder [27].

Consider first a situation without interactions as discussed previously in [7]. Figure 6 shows the ground state density for a condensate in the combined potential of the magnetic trap and the optical lattice. As expected the modulation of the density due to the lattice is visible. The addition of two very weak lattices at wavelengths of $\lambda=960$ and $1060 \mathrm{~nm}$ changes this situation drastically as shown in figure 7 . The exponential tails of the ensemble density are a clear manifestation of Anderson-like localization.

However, it is well known that localization phenomena are strongly influenced by the presence of interactions [28]. Therefore a simulation including these interactions is necessary to predict parameter regimes for the observation of localization phenomena. 


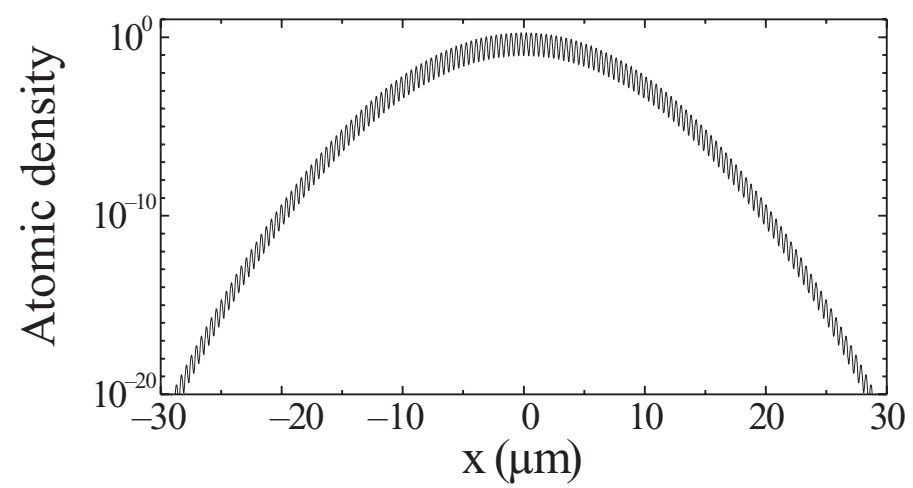

Figure 6. Ground state density of a non-interacting BEC in the combined potential of a magnetic trap $\left(\omega_{x}=2 \pi \times 4 \mathrm{~Hz}\right)$ and an optical lattice with a wavelength of $\lambda=825 \mathrm{~nm}$ and a depth of $6.5 E_{\mathrm{r}}$. Note the logarithmic vertical scale.

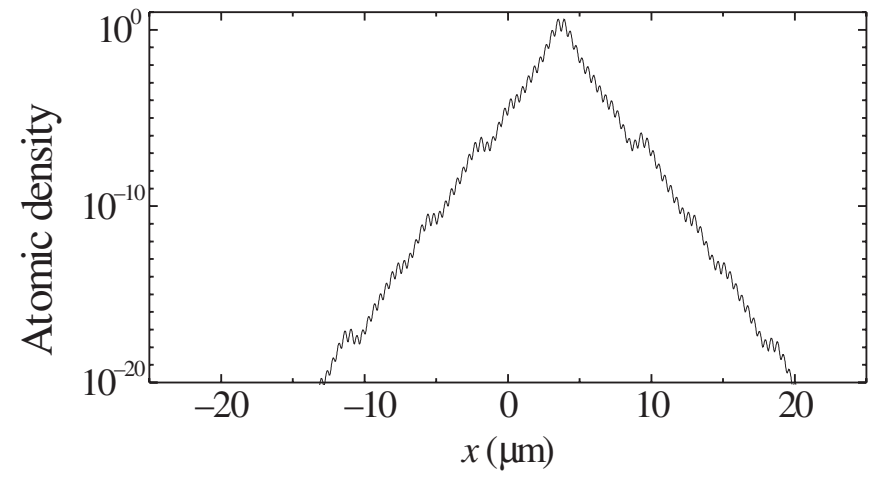

Figure 7. Ground state of a non-interacting BEC in the potential of figure 6 with two additional weak optical lattices at $\lambda=960$ and $1060 \mathrm{~nm}$ with depths of $0.2 E_{\mathrm{r}}$.

\subsection{Screening due to interaction}

Figure 8 shows the ground state solution of the GPE for a disordered potential that changes on the length scale of the optical lattice. While the effective mass approach can no longer be applied in this case, the localization signatures still do not emerge. This is due to the fact that the interaction between atoms delocalize the condensate.

The effect can be visualized by considering an effective potential. That is, once the solution $\phi_{0}$ of the stationary GPE is known, the equation can be considered as a Schrödinger equation

$$
-\frac{1}{2} \frac{\partial^{2} \phi_{0}(x)}{\partial x^{2}}+V_{\mathrm{eff}}(x) \phi_{0}(x)=\mu \phi_{0}(x)
$$

with the effective potential

$$
V_{\mathrm{eff}}(x)=\frac{x^{2}}{2}+V_{0} \cos ^{2}(k x)+V_{\mathrm{dis}}(x)+g\left|\phi_{0}(x)\right|^{2}
$$



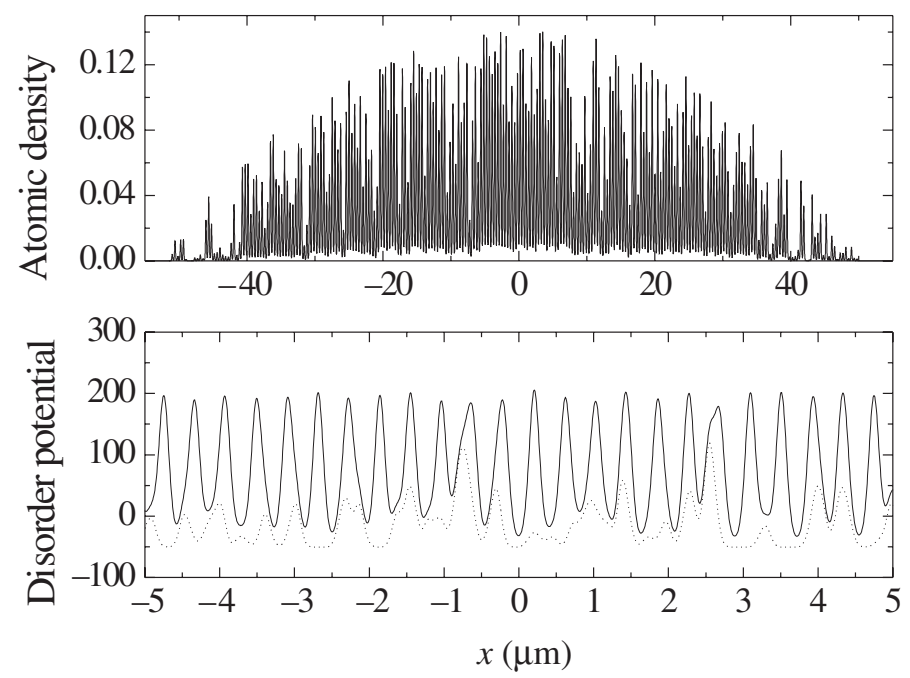

Figure 8. Top panel: ground state solution of the GPE for a condensate in the combined potential of the magnetic trap, optical lattice and disorder potential. Bottom panel: disorder potential $V_{\mathrm{dis}}(x)$ (dashed line) and disorder potential plus the $g\left|\phi_{0}(x)\right|^{2}$ term (solid line). The depth of the optical lattice is $6.5 E_{\mathrm{r}}$, while the depth of the disorder potential is $0.4 E_{\mathrm{r}}$. The coupling constant is $g=1800$.

As shown in figure 7, the situation without the nonlinear term $g\left|\phi_{0}(x)\right|^{2}$ leads to the observation of Anderson-like localization. However, when this term is present the effective potential loses its disordered nature due to a screening effect. Figure 8 shows the potentials $V_{\text {dis }}(x)$ and $V_{\text {dis }}(x)+g\left|\phi_{0}(x)\right|^{2}$. It is apparent that in the effective potential the disorder is smoothed and screened. For typical experimental parameters, the term $g\left|\phi_{0}(x)\right|^{2}$ dominates over $V_{\text {dis }}(x)$ and consequently the randomness necessary for localization is lost.

Hence a fine scale disorder alone is not sufficient to induce a non-trivial localization in the system. Additional control of the interaction is necessary. This effect is due to the accumulation of atoms in the wells of the random potential. In these regions of high density the nonlinear term in the GPE effectively smoothes the potential modulations [29]. A detailed discussion of this screening effect was recently given in [30].

\subsection{Analysis of localization}

We have investigated the transition from a fully delocalized to a localized ground state of a bosonic lattice gas in two ways. Firstly, a range of interaction strengths $g\left|\phi_{0}(x)\right|^{2}$ were investigated numerically for a fixed depth of the additional lattices. In current experiments a variation of the interaction strength can be realized by varying the density $\left|\phi_{0}(x)\right|^{2}$ of the sample or by using a Feshbach resonance to change the coupling constant $g$. Our main aim was to investigate if suitable parameters for the experimental observation of localization can be obtained. Secondly we have investigated the onset of localization as the disorder strength is increased in a weakly interacting BEC.

Our calculations were performed in an experimentally accessible regime with a trap frequency of $2 \pi \times 4 \mathrm{~Hz}$ and a pseudorandom potential equivalent to the one used for figure 7 . 


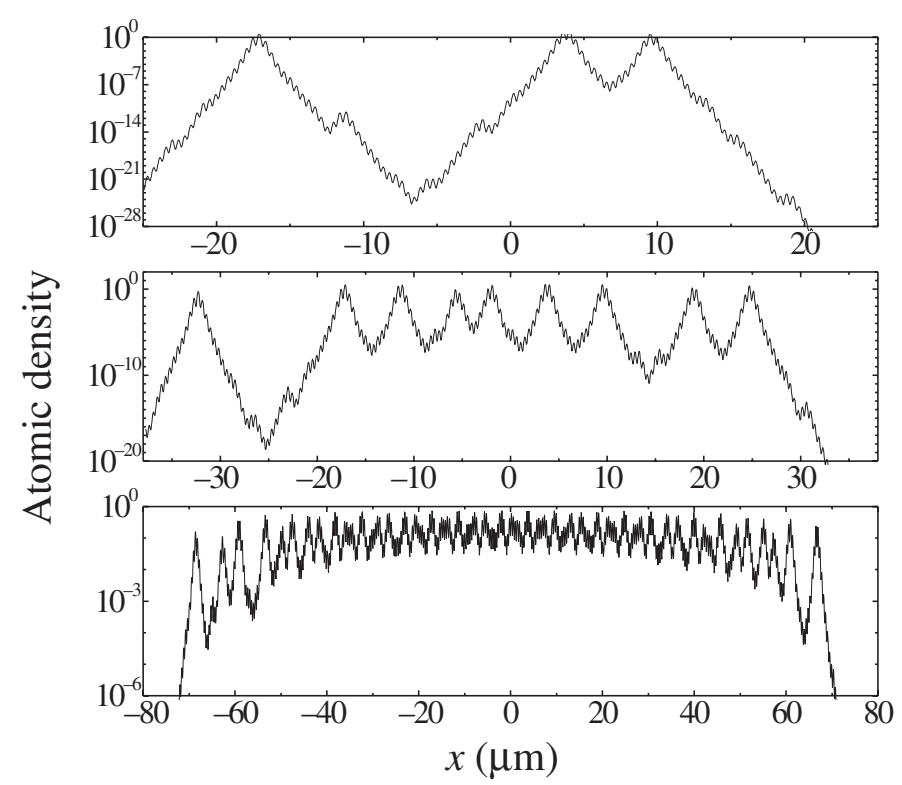

Figure 9. Ground states of the GPE for a condensate in the combined potential of the magnetic trap, optical lattice and pseudorandom potential. The depth of the optical lattice is $6.5 E_{\mathrm{r}}$, while the depths of the additional lattices forming the pseudorandom potential are $0.2 E_{\mathrm{r}}$. The coupling constants $g$ for the panels are: 0.5 (top), 8 (middle), 256 (bottom). Note the different scales in the panels.

For $g=0$, one obtains Anderson-like localization of the ground state wavefunction which is characterized by an exponential localization as shown in figure 7 .

Figure 9 shows ground states within this potential for three values of the interaction parameter. As $g$ is increased the number of localization centres grows and for large values of $g$ they overlap considerably. This behaviour suggests that the condensate wavefunction becomes a combination of these localized states due to nonlinear interactions. When $g$ is of the order of 500, one can no longer distinguish individual localized states and the clear signature of non-trivial localization vanishes.

The case of $g=256$ shown in figure 9 is equivalent to a 3D experimental realization with trap frequencies of $\omega_{x}=2 \pi \times 4 \mathrm{~Hz}$ and $\omega_{\perp}=2 \pi \times 40 \mathrm{~Hz}$ and $N=10^{4}$ atoms. The ground state simulation shows that characteristic features of Anderson-like localization are present, while the experimental parameters are within reach.

In a second part of our analysis we have investigated the localization length as a function of the disorder depth. The localization length $l$ was obtained by fitting the individual localization sites within the ground state density by $\left|\phi_{0}(x)\right|^{2} \propto \exp \left(-\left|x-x_{0}\right| / l\right)$.

Figure 10 shows these localization lengths for depths of the superlattice potential up to $0.2 E_{\mathrm{r}}$. The simulation was performed for interaction strengths of $g=0$ and $g=8$ in a trap with $\omega_{x}=2 \pi \times 4 \mathrm{~Hz}$ trapping frequency. Each point in the figure is the result of a fit to peaks in the wavefunctions depicted in figure 9 . Within the scope of our simulation the amplitude of the pseudorandom potential has to be sufficiently big to ensure that the localization length is smaller than the size of the system. 


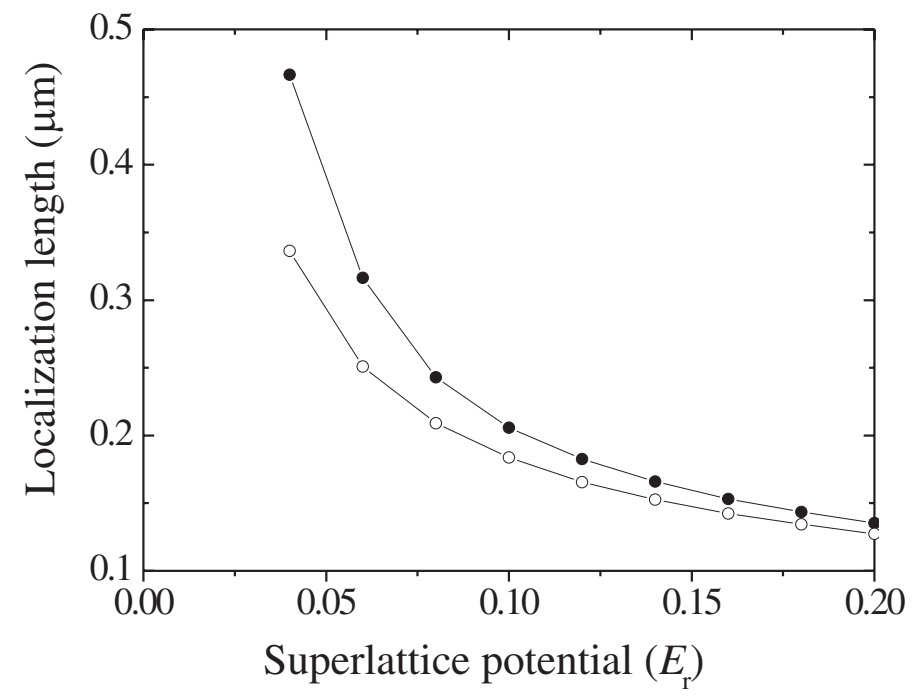

Figure 10. Localization length as a function of the depth of the additional optical lattice potentials for two interaction strengths $g=0(\circ)$ and $g=8(\bullet)$. The depth of the main optical lattice is $6.5 E_{\mathrm{r}}$.

This analysis clearly shows two distinct features of localization. Firstly, the localization length strongly depends on the depth of the superlattice. Even a very small added pseudorandom potentials leads to localization in small localization sites. This confirms the expected nonperturbative character of localization. Secondly the analysis shows that the interaction strength only has a small effect on the localization length in the strongly localized regime as shown in figure 10. This can also be inferred from figure 9 taking into account the different axis scales.

\subsection{Analysis of superfluidity}

To further investigate the effect of interactions we have analysed the superfluid fraction of the sample as a function of the coupling constant $g$ in a potential box.

The superfluid fraction is obtained by calculating the response of the condensed sample to twisted boundary conditions [31]. Within this model the superfluid fraction acquires additional kinetic energy due to a spatially varying phase. A comparison of the energy with and without phase twist yields the superfluid fraction which is defined as $f_{\mathrm{s}}=2\left(E_{0}(v)-E_{0}(0)\right) / N v^{2}$. Here $E_{0}(v)$ is the ground state energy when a velocity field $v$ is imposed on the system (i.e. we compute the ground state solution in the form $\phi_{0}(x) \exp (\mathrm{i} v x)$ where $\phi_{0}(x)$ fulfils periodic boundary conditions) [31]. In our calculations, the size of the potential box was chosen to match the size of the atomic cloud in the harmonic potential. It is important to note that this method only represents one possible definition of superfluidity [31, 32]. Within the GPE framework, it does not include higher excitation modes or atoms depleted from the condensate [10].

Figure 11 shows the superfluid fraction as a function of the coupling constant. In the presence of an optical lattice some loss of the superfluid fraction is observed, but even for small interaction strengths a considerable superfluid fraction remains. This behaviour drastically changes when the pseudorandom disorder created by the two additional optical lattices at 960 and $1060 \mathrm{~nm}$ is added. At low values of the coupling constant $g$ the superfluid fraction strongly decreases, 


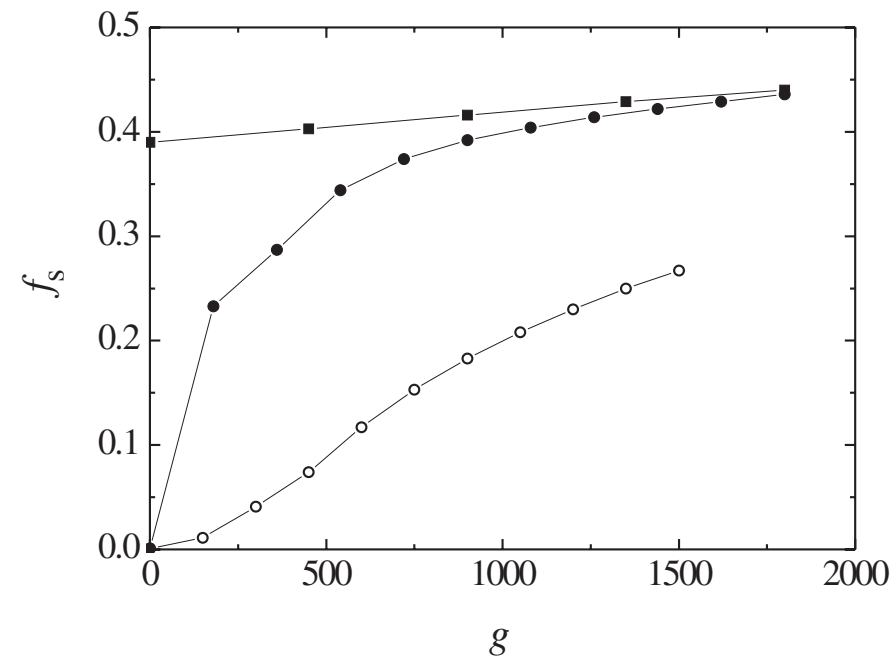

Figure 11. Superfluid fraction as a function of the coupling constant $g$ for a fixed superlattice depth of $0.2 E_{\mathrm{r}}$. Full circles $(\bullet)$ are obtained for a box potential corresponding to a trap frequency of $\omega_{x}=2 \pi \times 14 \mathrm{~Hz}$ and open circles (o) to $\omega_{x}=2 \pi \times 4 \mathrm{~Hz}$. Full squares ( $\left.\boldsymbol{\square}\right)$ show the case without a superlattice potential for $\omega_{x}=2 \pi \times 14 \mathrm{~Hz}$.

indicating the onset of localization. However, our analysis shows that the superfluid fraction remains large for the coupling constants $g$ in typical experimental realizations, indicating the absence of Anderson-like localization.

\subsection{Detection of localization}

The observation of features of Anderson-like localization may pose considerable experimental difficulties. Figure 12 shows the density distributions on a linear scale. In an experimental measurement the exponential nature of the density variation within the localization sites will probably not be visible. Most likely, limitations due to the imaging optics will inhibit the observation of individual localization sites for experimentally accessible densities.

However, the onset of localization leads to a considerable change in the ground state density when a small pseudorandom potentials is added. If the localization effect is indeed non-perturbative, it may be possible to detect this change of the density even for small added pseudorandom potentials.

A second avenue for the detection of localization is a time-of-flight measurement of the velocity distribution. The simulated density distribution after $20 \mathrm{~ms}$ of free expansion is shown in figure 13. Despite a clear difference in the ground state wavefunction, the width of the envelope of the zero-momentum peak is strikingly similar for all realizations. The results represent a clear distinction from the classical case, where the width of the expanded cloud depends strongly on the interaction parameter $g$. We conclude that the width of the zero-momentum peak mainly depends on the localization length $l$, which does not vary significantly as a function of $g$ in the simulations presented here.

These two options show a path towards the observation of Anderson-like localization in the regime discussed. 


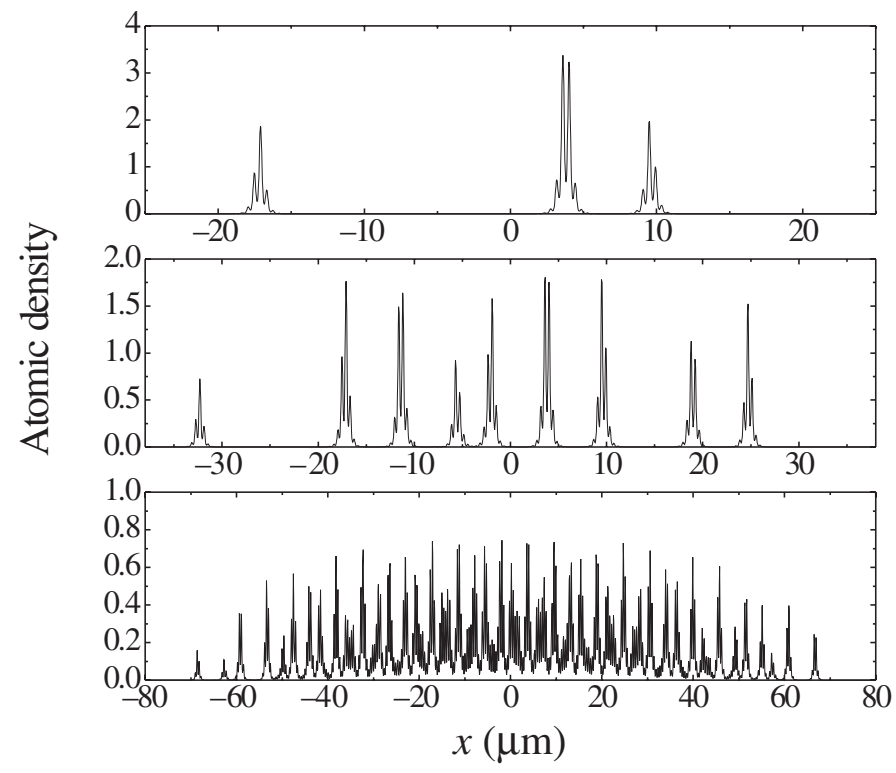

Figure 12. Ground states of the GPE (previously shown in figure 9 on a logarithmic scale) for a condensate in the combined potential of the magnetic trap, optical lattice and pseudorandom potential. The coupling constant $g$ for the panels are: 0.5 (top), 8 (middle), 256 (bottom).
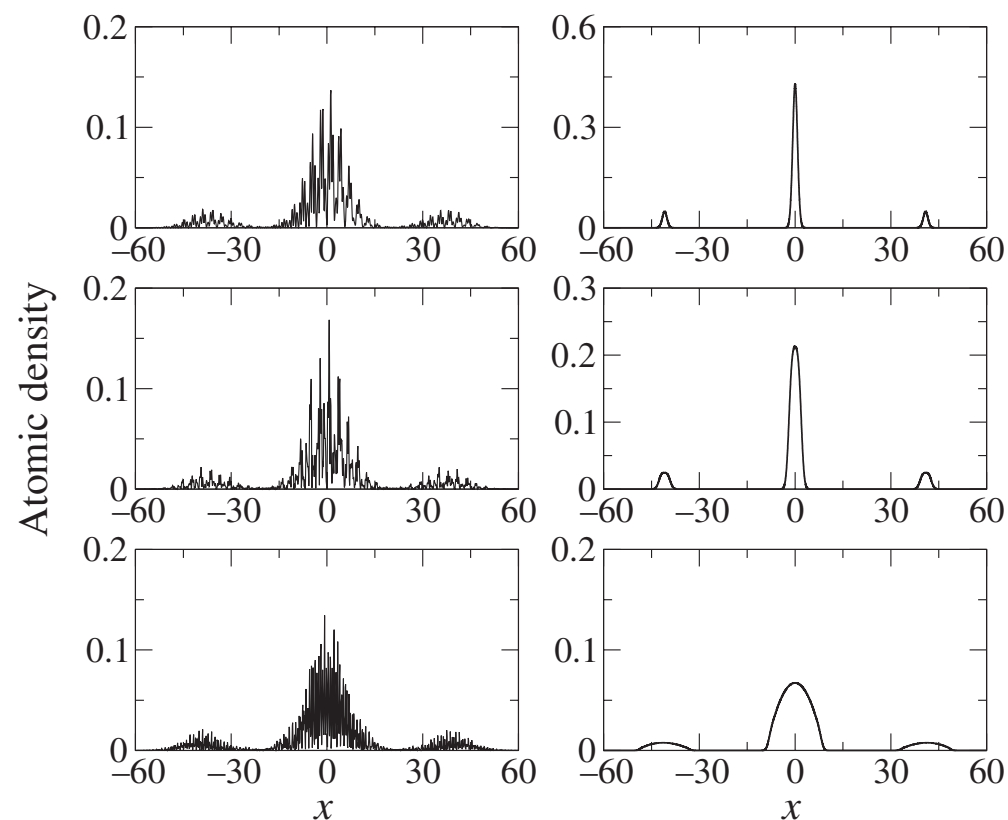

Figure 13. Atomic density after $20 \mathrm{~ms}$ of free expansion for a condensate prepared in the states shown in figure 12 (left column) and without disorder potential (right column). Oscillator units corresponding to a trap frequency of $2 \pi \times 4 \mathrm{~Hz}$ are used. 


\section{Conclusion}

We have presented a detailed theoretical analysis of two configurations for the production and observation of disordered quantum degenerate gases. This analysis showed that localization effects are not expected for the configurations involving a slowly varying disordered dipole potential. However, clear localization effects and a reduction of the superfluid fraction were observed when a superlattice was used to provide the disorder. These effects can be suppressed due to screening by nonlinear interactions within the sample. The dependence of the localization features on these interactions and on the depth of the superlattice potential were analysed in detail. It was shown that an analysis of the time-of-flight signal will allow for a conclusive evaluation of possible localization phenomena in the sample.

Within the experimental part a realization of a disordered lattice gas is described in detail. An analysis of the time-of-flight signal allows for a conclusive evaluation of possible localization phenomena in the sample. In accordance with the theoretical findings however, no localization effects are observed.

The theoretical work within this paper paves a clear path for the future observation of Anderson-like localization in cold atomic samples.

\section{Acknowledgments}

We thank L Santos, L Sanchez-Palencia and G V Shlyapnikov for fruitful discussions. We acknowledge support from the Deutsche Forschungsgemeinschaft (SFB 407, SPP 1116, GK 282, 436 POL), the ESP Programme QUDEDIS, the Polish government funds PBZ-MIN008/P03/2003 (KS) and 1P03B08328 (2005-08) (JZ).

\section{References}

[1] Anderson P W 1958 Phys. Rev. 1091492

[2] Lee P A and Ramakrishan R V 1985 Disordered electronic systems Rev. Mod. Phys. 57287 and references therein

[3] Kramer B and MacKinnon A 1993 Rep. Prog. Phys. 561469

[4] Wellens T, Grémaud B, Delande D and Miniatura C 2006 Phys. Rev. A 73013802

[5] Horak P, Courtois J-Y and Grynberg G 1998 Phys. Rev. A 583953

[6] Kuhn R C, Miniatura C, Delande D, Sigwarth O and Müller C A 2005 Phys. Rev. Lett. 95250403

[7] Damski B et al 2003 Phys. Rev. Lett. 91080403

[8] Diener R B et al 2001 Phys. Rev. A 64033416

[9] Roth R and Burnett K 2003 J. Opt. B: Quantum Semiclass. Opt. 5 S50

[10] Roth R and Burnett K 2003 Phys. Rev. A 68023604

[11] Bar-Gill N, Pugatch R, Rowen E, Katz N and Davidson N 2006 Preprint cond-mat/0603513

[12] Gavish U and Castin Y 2005 Phys. Rev. Lett. 95020401

Massignan P and Castin Y 2006 Phys. Rev. A 74013616

[13] Folman R et al 2002 Adv. At. Mol. Opt. Phys. 48263

Henkel C, Kruger P, Folman R and Schmiedmayer J 2003 Appl. Phys. B 76173

Wang D-W, Lukin M D and Demler E 2004 Phys. Rev. Lett. 92076802

[14] Lye J E, Fallani L, Modugno M, Weirsma D, Fort C and Inguscio M 2005 Phys. Rev. Lett. 95070401

[15] Fort C, Fallani L, Guarrera V, Lye J, Modugno M, Wiersma D S and Inguscio M 2005 Phys. Rev. Lett. 95170410 
[16] Clément D, Varon A F, Hugbart M, Retter J A, Bouyer P, Sanchez-Palencia L, Gangardt D M, Shlyapnikov G V and Aspect A 2005 Phys. Rev. Lett. 95170409

[17] Clement D, Varon A F, Retter J A, Sanchez-Palencia L, Aspect A and Bouyer P 2006 New J. Phys. 8165

[18] Modugno M 2006 Phys. Rev. A 73013606

[19] Schulte T, Drenkelforth S, Kruse J, Ertmer W, Arlt J, Sacha K, Zakrzewski J and Lewenstein M 2005 Phys. Rev. Lett. 95170411

[20] Fallani L, Lye J E, Guarrera V, Fort C and Inguscio M 2006 Preprint cond-mat/0603655

[21] Fisher M P A, Weichman P B, Grinstein G and Fisher D S 1989 Phys. Rev. B 40546

[22] Sanpera A, Kantian A, Sanchez-Palencia L, Zakrzewski J and Lewenstein M 2004 Phys. Rev. Lett. 93040401 Ahufinger V, Sanchez-Palencia L, Kantian A, Sanpera A and Lewenstein M 2005 Phys. Rev. A 72063616

Sanchez-Palencia L, Ahufinger V, Kantian A, Zakrzewski J, Sanpera A and Lewenstein M 2006 J. Phys. B: At. Mol. Opt. Phys. 39 S121-S134

[23] Ospelkaus C, Ospelkaus S, Humbert L, Ernst P, Sengstock K and Bongs K 2006 Preprint cond-mat/0607581

[24] Castin Y and Dum R 1996 Phys. Rev. Lett. 775315

[25] Pedri P et al 2001 Phys. Rev. Lett. 87220401

[26] Steel M J and Zhang W 1998 Preprint cond-mat/9810284

Pu H et al 2003 Phys. Rev. A 67043605

[27] Sokoloff J B 1985 Phys. Rep. 126189

[28] Scalettar R T, Batrouni G G and Zimanyi G T 1991 Phys. Rev. Lett. 663144

[29] Singh K G and Rokhsar D S 1994 Phys. Rev. B 499013

Rasmussen K O, Cai D, Bishop A R and Grønbech-Jensen N 1999 Europhys. Lett. 47421

Lee D K K and Gunn J M F 1990 J. Phys. C: Solid State Phys. 27753

[30] Sanchez-Palencia L 2006 Preprint cond-mat/0609036

[31] Lieb E H, Seiringer R and Yngvason J 2002 Phys. Rev. B 66134529

[32] Prokof'ev N V and Svistunov B V 2000 Phys. Rev. B 6111282 